\title{
Awaking the vacuum in relativistic stars
}

\author{
William C. C. Lima,, , George E. A. Matsas,, , 田 and Daniel A. T. Vanzella ${ }^{1, \text { 目 }}$ \\ ${ }^{1}$ Instituto de Física de São Carlos, Universidade de São Paulo, \\ Caixa Postal 369, 15980-900, São Carlos, SP, Brazil \\ ${ }^{2}$ Instituto de Física Teórica, Universidade Estadual Paulista, \\ Rua Dr. Bento Teobaldo Ferraz, 271 - Bl. II, 01140-070, São Paulo, SP, Brazil
}

(Dated: April 27, 2018)

\begin{abstract}
Void of any inherent structure in classical physics, the vacuum has revealed to be incredibly crowded with all sorts of processes in relativistic quantum physics. Yet, its direct effects are usually so subtle that its structure remains almost as evasive as in classical physics. Here, in contrast, we report on the discovery of a novel effect according to which the vacuum is compelled to play an unexpected central role in an astrophysical context. We show that the formation of relativistic stars may lead the vacuum energy density of a quantum field to an exponential growth. The vacuumdriven evolution which would then follow may lead to unexpected implications for astrophysics, while the observation of stable neutron-star configurations may teach us much on the field content of our Universe.

PACS numbers: 04.40.Dg, 04.62.+v, 95.30.Sf
\end{abstract}

Born from the efforts to formulate a consistent relativistic version of quantum mechanics, the successful formalism of quantum field theory (QFT) has unveiled an incredibly rich structure for what was thought in classical physics to be the most uninteresting of the states: the vacuum. From the early Dirac sea of negative-energy states to the picture of virtual particles constantly being created and annihilated, the vacuum has acquired conceptual importance for a consistent description of nature. However, from the observational point of view, its existence continues to be almost as evasive as in classical physics, demanding carefully designed experiments in order to detect its subtle effects. (The Casimir effect [1], according to which two (or more) objects experience a force between them solely due to the fact that their presence changes the vacuum energy in a position-dependent way, is such an example, successfully observed in laboratory. See, e.g., Refs. 2, 3].) Particularly interesting is the fact that the vacuum, being a dynamical entity, gravitates, which using the concepts of general relativity (GR) means that it affects and is affected by the geometry of the spacetime. This rests at the root of the Hawking effect [4, 5], according to which black holes should emit a thermal bath of particles. Although this semiclassicalgravity effect of "black hole evaporation" has become a rare landmark for those who seek a complete quantum gravity theory, it is virtually unobservable for realistic astrophysical black holes. Here, in contrast, we investigate possible astrophysical implications of a recently-proposed semiclassical-gravity mechanism [6] by which the vacuum of free quantum fields is forced to play a central role. We focus on the context of relativistic stars and show that the formation of (realistic) compact objects may disturb the vacuum of a quantum field in a way which leads its energy density to an exponential growth. Eventually, this vacuum energy should take control over the evolution of the background spacetime, determining the ultimate fate of the relativistic star and possibly leading to unexpected implications for astrophysics. On the other hand, the observation of stable neutron-star configurations may be used to rule out the existence of fields for which the effect should have been triggered.

Vacuum energy in gravitational fields: The task of calculating "vacuum energies" of quantum fields in gravitational backgrounds is one which is properly addressed (though not easily performed in general) only by using techniques of quantum field theory in curved spacetimes (QFTCS), which is the minimal and most conservative way of merging the successful formalisms of QFT and GR [7-12]. Born more than four decades ago [7, 8], QFTCS has proven its value by providing the proper context in which legitimate "low-energy" (i.e., below Planck scale) quantum gravitational phenomena, like the black hole evaporation, could be unveiled. Therefore, before presenting the arguments which lead to our main result, let us give the reader a brief overview on how to obtain the vacuum energy of a quantum field in a curved background using the canonical approach to QFTCS. In a nutshell, it goes as follows: (i) one solves the generally covariant field equation for a complete set of normal modes $\left\{u_{\alpha}^{(+)}, u_{\alpha}^{(-)}\right\}$of the field $\Phi$ in a given fixed background geometry with appropriate boundary/initial conditions which express the choice of the (Fock) Hilbert space of interest; (ii) one (formally) expands the field operator $\hat{\Phi}$ using these normal modes with the operator coefficients of the positive-norm $u_{\alpha}^{(+)}$(negative-norm $u_{\alpha}^{(-)}$) modes being interpreted as the annihilation (creation) operators for that particular mode; (iii) then one (formally) constructs the stress-energy-tensor operator $\hat{T}_{\mu \nu}$ out of its classical expression by substituting $\Phi$ by $\hat{\Phi}$, and calculates its expectation value in the state of interest (usually the vacuum of the chosen Fock Hilbert space). Since $\hat{T}_{\mu \nu}$ is a quadratic expression in $\hat{\Phi}$ (which should be viewed 
as an operator-valued distribution), some regularization and renormalization procedure must be applied in step (iii) in order to give rise to an expectation value which is free from ultraviolet divergences.

Because the background spacetime has to be fixed already in step (i), the procedure outlined above is expected to give meaningful results whenever the backreaction of the expectation value of $\hat{T}_{\mu \nu}$ on the background spacetime (through the semiclassical Einstein equations) can be neglected. Notwithstanding, failure to comply with this condition can be used to expose situations where semiclassical-gravity effects become crucial. This is the strategy we follow here.

Disturbing the vacuum during the formation of a star: For the sake of simplicity, we follow Ref. 6] and consider a real scalar field $\Phi$ with mass $m \geq 0$ and arbitrary coupling $\xi$ with the Ricci scalar curvature $R$. The field equation is the usual Klein-Gordon equation with the additional scalar-curvature coupling: $\left(-\square+m^{2}+\xi R\right) \Phi=0$. (We adopt natural units in which $\hbar=c=1$ unless stated otherwise.) We consider the case in which matter initially scattered throughout space with very low density eventually collapses to form a static (and stable) spherically symmetric compact object (according to classical GR). Therefore, the background metric can be taken to be asymptotically flat in the past and asymptotically static and spherically symmetric in the future:

$$
d s^{2} \sim\left\{\begin{array}{l}
-d t^{2}+d \vec{x}^{2} \\
f\left(-d t^{2}+d \chi^{2}\right)+r^{2}\left(d \theta^{2}+\sin \theta^{2} d \varphi^{2}\right), \text { past } \text { future }
\end{array}\right.
$$

where $f=f(\chi)>0$ and $r=r(\chi) \geq 0$ are functions of the coordinate $\chi$ alone, with $f(\chi) \rightarrow 1$ and $r(\chi) / \chi \rightarrow 1$ for $\chi \rightarrow \infty$, and $d r / d \chi>0$ so that no trapped light-like surface is present.

The natural in-vacuum state is the one associated with the normal modes $u_{\vec{k}}^{(+)}$and $u_{\vec{k}}^{(-)} \equiv\left(u_{\vec{k}}^{(+)}\right)^{*}$ which in the asymptotic past take the form of the usual flat-space stationary modes:

$$
u_{\vec{k}}^{(+)} \stackrel{\text { past }}{\sim}\left(16 \pi^{3} \omega_{\vec{k}}\right)^{-1 / 2} e^{-i\left(\omega_{\vec{k}} t-\vec{k} \cdot \vec{x}\right)},
$$

where $\vec{k} \in \mathbb{R}^{3}$ and $\omega_{\vec{k}}:=\sqrt{\vec{k}^{2}+m^{2}}$ is interpreted as the energy of the mode. Since the spacetime is also asymptotically static in the future, after the compact object is formed, there is a natural out-vacuum state, which is the one associated with a different set of normal modes $v_{\varpi l \mu}^{(+)}$and $v_{\varpi l \mu}^{(-)} \equiv\left(v_{\varpi l \mu}^{(+)}\right)^{*}$ which in the asymptotic future assume the stationary form

$$
v_{\varpi l \mu}^{(+)} \stackrel{\text { future }}{\sim}[\sqrt{2 \varpi} f(\chi) r(\chi)]^{-1} e^{-i \varpi t} F_{\varpi l}(\chi) Y_{l \mu}(\theta, \varphi),
$$

where $Y_{l \mu}(l=0,1,2, \ldots$ and $\mu=-l, \ldots, l)$ are the usual spherical-harmonic functions and $F_{\varpi l}$ are solutions of Schrödinger-type equations

$$
\left(-d^{2} / d \chi^{2}+V_{\mathrm{eff}}^{(l)}\right) F_{\varpi l}=\varpi^{2} F_{\varpi l}
$$

satisfying usual boundary conditions and normalization. Here, $\varpi$ is the energy of the mode (which no longer needs to be greater than $m$ ) according to static observers in the asymptotic future which are far away from the compact object. The effective potential $V_{\mathrm{eff}}^{(l)}=V_{\mathrm{eff}}^{(l)}(\chi)$ is given by

$$
V_{\mathrm{eff}}^{(l)}=f\left[m^{2}+\xi R+l(l+1) / r^{2}\right]+(1 / r) d^{2} r / d \chi^{2} .
$$

The functions $f(\chi)$ and $r(\chi)$ can be related to the matter/energy distribution of the compact object through the Einstein equations of GR. For instance, assuming the compact object to be made of perfect fluid (assumption which will be used throughout this article for simplicity), the effective potential can be put into the form

$$
V_{\mathrm{eff}}^{(l)}=f\left[m^{2}+(\xi-1 / 6) R+\frac{l(l+1)}{r^{2}}+\frac{8 \pi G}{3}(\bar{\rho}-\rho)\right],
$$

where $\rho=\rho(\chi)$ is the matter/energy density of the compact object and $\bar{\rho}=\bar{\rho}(\chi)$ represents its averaged value up to $\chi: \bar{\rho}(\chi):=3 M(\chi) /\left(4 \pi r(\chi)^{3}\right)$, with $M(\chi)$ being the mass of the object up to $\chi$. (Recall also that, according to GR, $R=8 \pi G(\rho-3 p)$, where $p$ is the pressure which sustains the configuration of the compact object and $G$ is Newton's gravitational constant.)

In general the in- and out-vacuum do not coincide, which means that particles are created due to the change in the gravitational background. However, this particle creation is usually negligible. In contrast, as recently pointed out in a quite general context [6], there is another possible influence of the gravitational background on the quantum vacuum: well-behaved gravitational fields may trigger an exponential increase of the vacuum energy density leading to a vacuum-dominated scenario. Applied to the context of compact objects, the gravitational fields which are entitled to "awake" the vacuum energy are the ones for which the effective potential in Eq. (6) gets sufficiently negative somewhere in the asymptotic future so that Eq. (4) possesses well-behaved solutions $F_{\varpi l}$ for $\varpi^{2}=-\Omega^{2}<0$. In this case, additional, non-stationary, normal modes $w_{\Omega l \mu}^{(+)}$and $w_{\Omega l \mu}^{(-)} \equiv\left(w_{\Omega l \mu}^{(+)}\right)^{*}$ with the asymptotic behavior

$$
w_{\Omega l \mu}^{(+)} \stackrel{\text { future }}{\sim} \frac{\left(e^{-\Omega t+i \pi / 12}+e^{\Omega t-i \pi / 12}\right)}{\sqrt{2 \Omega}} \frac{F_{\varpi l}(\chi)}{r(\chi)} Y_{l \mu}(\theta, \varphi)
$$

are necessary in order to expand the field in the asymptotic future. This implies that at least some of the in-modes eventually go through a phase of exponential growth, which leads to an exponential increase of the vacuum expectation value of $\hat{\Phi}^{2}$. This is then reflected in an exponential growth of the vacuum expectation value of $\hat{T}_{\mu \nu}$ (see Eqs. (10-13) of Ref [6]). (It is important to note that this asymptotic divergence has nothing to do with high-frequency modes, so that renormalization plays no important role in this analysis.) As a consequence, no matter what the value of the classical energy density of 
the compact object is, eventually the vacuum energy density (and pressure, and momentum density) of the field will dominate and take control over the spacetime evolution.

Sharp transition to vacuum dominance in relativistic stars: It is interesting to note that in principle the vacuum-dominance effect could be triggered for massive fields with $m^{2} \ll R \sim G \rho$ (so that a region where $V_{\text {eff }}^{(l)}$ is negative might exist for values of $|\xi|$ not too large). However, recovering the units in which these quantities are usually expressed, this condition implies

$$
\frac{m^{2} /\left(3.5 \times 10^{-12} \mathrm{eV}\right)^{2}}{\rho /\left(10^{14} \mathrm{~g} / \mathrm{cm}^{3}\right)} \ll 1 .
$$

Therefore, considering that relativistic stars can reach densities $\rho \sim 10^{14-17} \mathrm{~g} / \mathrm{cm}^{3}$ [13], the most "natural" mass satisfying this is $m=0$, which we shall assume in what follows.

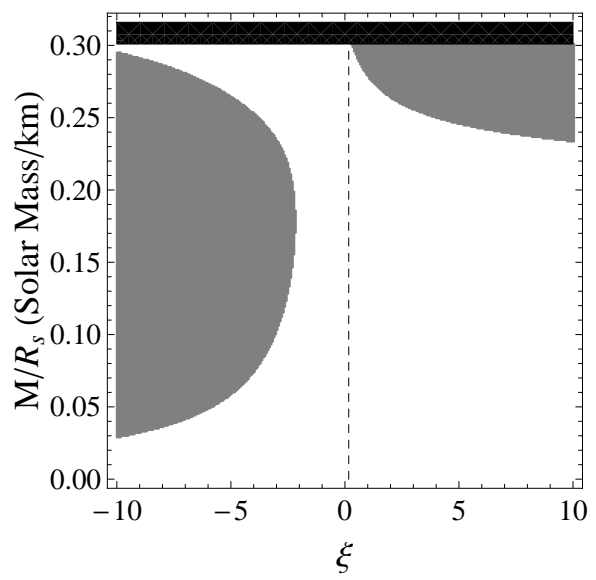

FIG. 1: Diagram showing the values of the ratio $M / R_{s}$ of uniform-density compact objects which trigger the exponential growth of the vacuum energy density of massless scalar fields with coupling $\xi$ (dark-gray region). The vertical dashed line indicates the conformal-coupling value $\xi=1 / 6$. The black region represents the values of $M / R_{s}$ for which no equilibrium can be classically obtained.

It may come as a surprise to verify that the simplest distribution possible for an idealized compact object, the uniform energy-density distribution, is already entitled to awake the vacuum energy of quantum fields. Assuming $\rho=\bar{\rho} \equiv \rho_{0}$, the Tolman-Oppenheimer-Volkoff (TOV) equation of relativistic hydrostatic equilibrium can be analytically integrated to obtain the pressure distribution $p$ and then the function $f$ (see, e.g., Ref. [14]). Then, substituting these into Eq. (6), a numerical search for "bound" solutions of Eq. (4) with $\varpi^{2}<0$ (and $l=0$, which is the most promising case) can be performed for several values of the radius $R_{s}$ and the mass $M=4 \pi \rho_{0} R_{s}^{3} / 3$ of the compact object. In this uniformdensity case, the existence of such "bound" solutions depends only on the ratio $M / R_{s}$ and the value of $\xi$. The

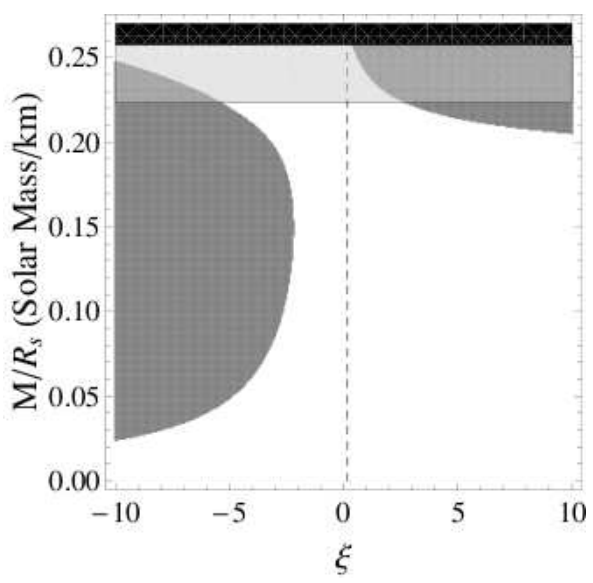

FIG. 2: Same as diagram in Fig. 11 but now for compact objects with parabolic density profile. The light-gray region represents values of $M / R_{s}$ for which equilibrium configurations are classically unstable for most realistic equations of state (see, e.g., Ref. [15]).

results of this search are shown in Fig. 1. We see that a massless field with any value of $\xi>1 / 6$ or $\xi \lesssim-2$ can have its vacuum energy density exponentially amplified for some range of $M / R_{s}$.

This idealized uniform-density case serves to illustrate that well-behaved background geometries can indeed induce the vacuum-dominance effect for fields with appropriate masses and scalar-curvature couplings. This, however, is not enough if we want to explore possible observational implications. For that matter, more realistic density and pressure profiles for the compact object have to be used. Although there is a certain amount of uncertainty on the correct equation of state for matter at densities as high as $10^{15-17} \mathrm{~g} / \mathrm{cm}^{3}$, the density profile of realistic neutron stars with masses $1.5 M_{\odot} \sim 2.2 M_{\odot}$ can be closely approximated by a parabolic radial dependence, $\rho=\rho_{c}\left(1-r^{2} / R_{s}^{2}\right)$ [15], where $\rho_{c}=15 M /\left(8 \pi R_{s}^{3}\right)$ is the central density. In this case, the TOV equation can also be analytically integrated to obtain the pressure distribution $p$ and the background metric [16], and a numerical search for "bound" solutions of Eq. (4) with $\varpi^{2}<0$ (and $l=0$ ) can be performed, as in the previous case. The existence of "bound" solutions again depends only on the ratio $M / R_{s}$ and the value of $\xi$. The results of this search are shown in Fig. 2. Note that Figs. 1 and 2 are very similar but it is worthwhile to point out that in this more realistic case the effect is triggered for smaller values of $M / R_{s}$. In particular, values of $M / R_{s}$ for which most realistic equations of state are causal [15], and which represents classically stable configurations, can awake the vacuum of fields with proper couplings.

The time scale which governs the exponential growth of the vacuum energy density, energy flow and pressure is given by $\Omega^{-1}$ (which usually is of order $\left|V_{\mathrm{eff}}^{(l)}\right|^{-1 / 2}$ ). In fact, the dominant contribution to the vacuum energy 
density in the asymptotic future can be estimated from Ref. [6]:

$$
\begin{aligned}
\rho_{V} & \stackrel{\text { future }}{\sim} \Omega h(\bar{r}) e^{2 \Omega t} / R_{s}^{3} \\
& \sim h(\bar{r}) \exp \left(\frac{t /\left(10^{-5} \mathrm{~s}\right)}{R_{s} /(10 \mathrm{~km})}\right) \frac{10^{-62} \mathrm{~g} / \mathrm{cm}^{3}}{R_{s}^{4} /(10 \mathrm{~km})^{4}}
\end{aligned}
$$

where $h(\bar{r})$ is a dimensionless function of $\bar{r}:=r / R_{s}$ and we have used that for a bound solution $\Omega \sim 1 / R_{s}$. The exact values assumed by $h(\bar{r})$ depend on the details of the spacetime evolution between the two static asymptotic regions, but for $\bar{r} \sim 1$ its order is not far from unit (though it is exponentially damped for large values of $\bar{r}$ ). Therefore, for a compact object with radius $R_{s} \approx 10 \mathrm{~km}$ whose effective potential happens to possess a bound solution, it would take only a few milliseconds (according to far away observers) for the vacuum energy density to become overwhelmingly dominant over classical matter densities as high as $10^{14-17} \mathrm{~g} / \mathrm{cm}^{3}$. This suggests that the transition from classical to vacuum dominance can be very sharp. In spite of this, we emphasize that the total vacuum energy is conserved (as it should in this static scenario where backreaction is not considered) due to the fact that the volume integral of $h(\bar{r})$ vanishes.

Final Comments: Although we have been talking about "vacuum dominance" of quantum fields, we would like to clarify that the appearance of exponentially growing modes already occurs in the classical level. This is evident from our argument that the modes given in Eq. (7) are necessary in order to expand an arbitrary (classical) field configuration. Therefore, the very same situations which trigger the exponential growth of the vacuum energy density will most likely lead to an exponential growth of the classical energy density stored in an initially non-vanishing field configuration. Here, however, we have opted to focus on the quantum version of the effect since it is triggered even in the absence of any initial classical perturbation, which makes its consequences more robust than the ones coming from its classical counterpart. The existence of initial classical perturbations can change the details but not the conclusion about whether or not the compact object will face the exponential instability, which is the main issue here.

The vacuum-awakening effect unveils a quite interesting interplay between semiclassical gravity and observational astrophysics. Concerning field theorists, the observation of some relativistic stars may be used to rule out the existence of certain fields in Nature. For instance, according to our Fig. 22 the observation of a stable and approximately spinless cold neutron star with mass $M$ and radius $R_{s}$ (with an approximately parabolic density profile) rules out the existence of massless scalar fields with couplings $\xi$ for which $\left(\xi, M / R_{s}\right)$ lie in the darkgray region. Considering that $95 \%$ of the energy content of the Universe is unknown, finding ways of testing the existence of free fields (for which there would be no rea- son to assume an initial classical perturbation) is very welcome. Finally, concerning astrophysicists, the awakening of the vacuum energy of certain fields may determine the ultimate fate of some relativistic stars. This will eventually depend on how the vacuum energy backreacts on the spacetime, as ruled by the semiclassical Einstein equations. The vacuum-driven evolution may lead, e.g., to (i) a complete collapse, hiding the negativeeffective-potential region inside an event horizon, or (ii) an explosion, ejecting mass and bringing the effective potential back to a profile admitting no bound solution (or possibly a combination of both). Only a detailed numerical analysis, beyond the scope of this article, can decide what will happen once backreaction is taken into account. Either way, we would have an unusual situation where the vacuum would be responsible for an event of astrophysical proportions.

The authors acknowledge partial financial support from Fundação de Amparo à Pesquisa do Estado de São Paulo (FAPESP) and Conselho Nacional de Desenvolvimento Científico e Tecnológico (CNPq). We also thank the anonymous referee for his/her valuable comments which helped to improve this version of the manuscript.

* Electronic address: william@ursa.ifsc.usp.br

$\dagger$ Electronic address: matsas@ift.unesp.br

¥ Electronic address: vanzella@ifsc.usp.br

[1] H. B. G. Casimir, Proc. K. Ned. Akad. Wet. 51, 793 (1948).

[2] H. B. Chan, V. A. Aksyuk, R. N. Kleiman, D. J. Bishop, and F. Capasso, Science 291, 1941 (2001).

[3] S. K. Lamoreaux, Rep. Prog. Phys. 68, 201 (2005).

[4] S. W. Hawking, Nature 248, 30 (1974).

[5] S. W. Hawking, Commun. Math. Phys. 43, 199 (1975).

[6] W. C. C. Lima and D. A. T. Vanzella, Phys. Rev. Lett. 104, 161102 (2010).

[7] L. Parker, Phys. Rev. Lett. 21, 562 (1968).

[8] L. Parker, Phys. Rev. 183, 1057 (1969).

[9] N. D. Birrell and P. C. W. Davies, Quantum Fields in Curved Space (Cambridge Univ. Press, Cambridge, 1982).

[10] S. A. Fulling, Aspects of Quantum Field Theory in Curved Spacetime (Cambridge Univ. Press, Cambridge, 1989).

[11] R. M. Wald, Quantum Field Theory in Curved Spacetime and Black Hole Thermodynamics (Univ. of Chicago Press, Chicago, 1994).

[12] L. Parker and D. Toms, Quantum Field Theory in Curved Spacetime: Quantized Fields and Gravity (Cambridge Univ. Press, Cambridge, 2009).

[13] J. M. Lattimer and M. Prakash, Phys. Rep. 442, 109 (2007).

[14] R. M. Wald, General Relativity (Univ. of Chicago Press, Chicago, 1984).

[15] J. M. Lattimer and M. Prakash, Astrophys. J. 550, 426 (2001).

[16] R. C. Tolman, Phys. Rev. 55, 364 (1939). 